\title{
Colliders race for the Higgs
}

\section{JACKSONVILLE, FLORIDA}

Soon after the news emerged on 29 March that a magnet test in the Large Hadron Collider (LHC) atom-smasher had failed, two physics bloggers decided to have a bit of fun with the idea. On 1 April they posted blogs announcing news such as 'Three years of delay for LHC start-up'. Visitors found the reports all too easy to swallow, decrying it as a cruel blow. Only then did one blogger add an April Fool's spoiler, with an apology: "Sorry to those of you who got hurt by not understanding that in the first place."

If these blog postings were close to the bone for many, it is because the LHC is running hard up against its deadline to switch on later this year. Conspiracy theories were also quick to fly: any postponement at the LHC, near Geneva, Switzerland, means that the Tevatron collider at the Fermi National Accelerator Laboratory in Illinois has more time to discover the last part of the standard model of particle physics - the Higgs boson.

On 15 April, the race became even more heated. At a meeting of the American Physical Society in Jacksonville, Florida, Tevatron physicists announced refined measurements for the masses of two particles that, taken together, lower the expected mass of the Higgs. The new estimates - of the mass of the top quark and of the W boson - put an upper limit on the Higgs mass of $144 \mathrm{GeV}$. In January, the best estimate was $153 \mathrm{GeV}$ (see Nature 445, 239; 2007). The lighter the Higgs, the better the chance the Tevatron has to detect it before it shuts down in 2009.

Researchers are more immediately worried about potential LHC delays caused by the failure of the magnet, which was supplied by Fermilab. "This does add to the burden of everything that has to be done before the machine switches on," says Pier Oddone, director of Fermilab. "We are embarrassed we created this additional problem." At the same time, some LHC researchers admit privately
"The magnet problem is a very small part of a bigger picture."

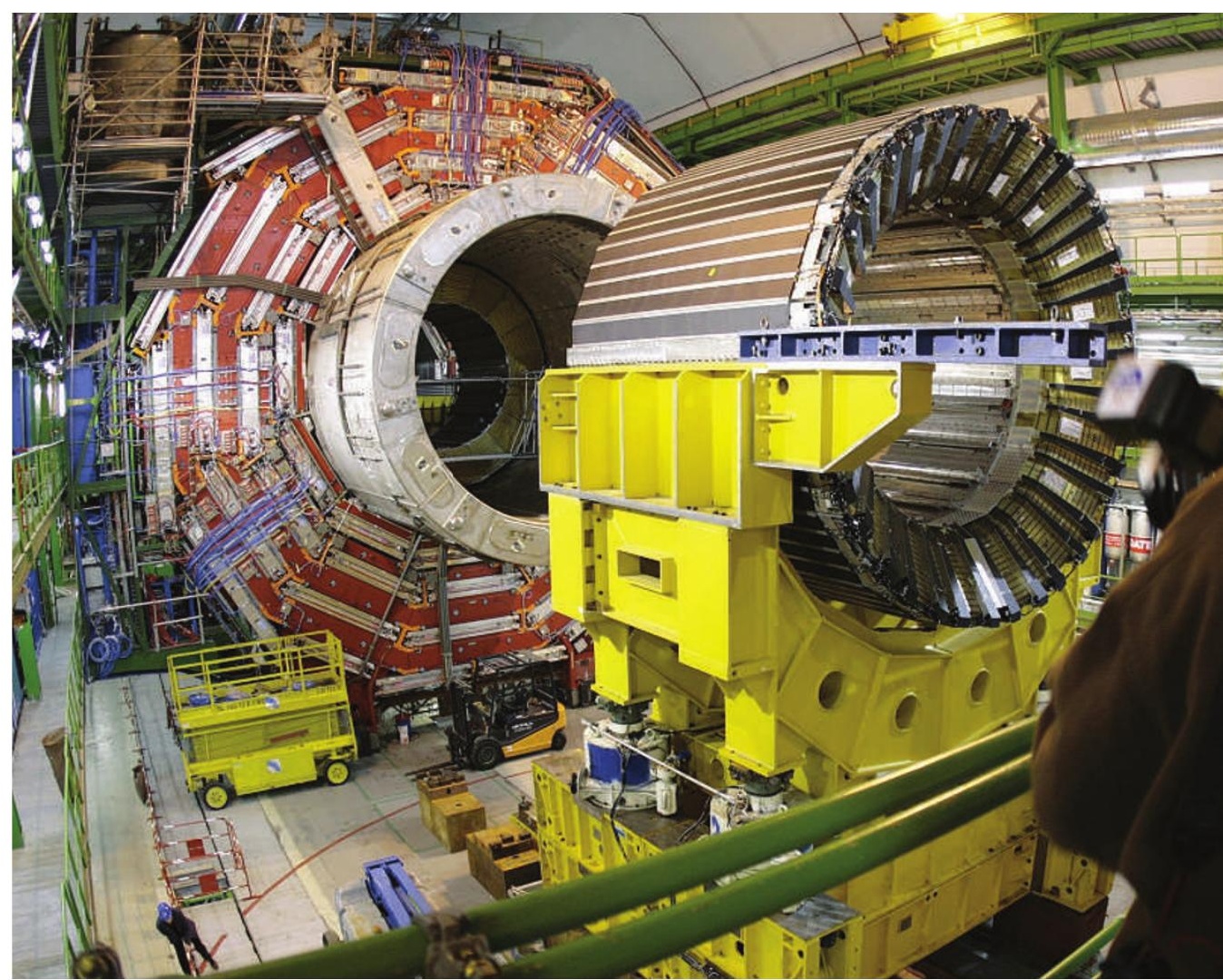

Big bang: the experiments at Europe's Large Hadron Collider are unlikely to be running before 2008.

The accident was not the first magnet failure: another Fermilab-supplied magnet had a faulty heat exchanger that had to be replaced a few months earlier. The second problem was one too many for Oddone, who has initiated an external review to figure out how the team missed such simple design flaws.

Meanwhile, CERN - the particle-physics laboratory in which the LHC is housed - and Fermilab are working together to find a fix and to minimize the effect on the LHC schedule. Options that the mistake might give them some breathing room.

The magnet that failed was part of an inner triplet designed to focus the proton beams before they interact. During a pressure test to simulate conditions expected in the collider, the eighttonne magnet jumped 13 centimetres, rupturing a pipe and causing a loud bang. The problem was quickly identified as a weakness in the supporting structure. "There was a definite oversight here," admits Stephen Holmes, head of accelerator physics at Fermilab. include reinforcing the triplet support structure with rods, or building physical buffers so that the magnets cannot move too far. Engineers hope to announce a solution by 25 April, and to test it on another triplet in the tunnel in early June.

The time needed to repair the magnets will need to be factored into the overall LHC operation, which was already running five weeks behind schedule before the accident, says project manager Lyndon Evans. There is also pressure to get the repair right the first time around, because there are few spare magnets available.

The most likely victim of any slippage is the first engineering run, planned for late 2007. The LHC is being chilled down in sections; it took from January to March 2007 to cool the first eighth of the machine to 1.9 kelvin. The process will become quicker, but cooling the final sectors in just two weeks each - as Evans says the current schedule demands - will be challenging, with or without the magnet repairs. "In my view the magnet problem has been blown out of proportion," he says. "It is a very small part of a bigger picture."

Evans adds that he still hasn't given up on the LHC conducting its first run in 2007, but admits that only an extreme optimist would share his view. With delay building on delay, a formal announcement of when the LHC might come online isn't expected until mid-May. And with CERN closing as it usually does for the winter months, no run in 2007 could push the first science run later than planned in 2008. And by that point, the Tevatron will have had another year or more to hunt for the Higgs. Sarah Tomlin 